\title{
Knee Myofascial Pain and Knee-Abdomen Syndromes; A Newly Identified Knee Disorders, Commonly Misdiagnosed as Arthritis, Multiple Case Study
}

\author{
Ibrahim Tobba Mohamed ${ }^{1}$, Olfat A Kandil ${ }^{* 2}$ \\ ${ }^{1}$ Assistant lecturer of orthopaedic physical therapy, Faculty of Physical Therapy, Beni-Suef University \\ ${ }^{2}$ Professor of biomechanics, Faculty of Physical Therapy, Beni-Suef University \\ *Corresponding author: Olfat A Kandil; dro_kandil@yahoo.com
}

Received 20 January 2022;

Accepted 13 February 2021;

Published 16 February 2022

\begin{abstract}
Background: Knee osteoarthritis is a common orthopedic condition. Imaging based pathoanatomical findings are utilized as a cornerstone for diagnosis of the condition, $97 \%$ of asymptomatic knees demonstrate pathoanatomical findings, causing doubt of diagnosis and efficiency of intervention based on asymptomatically present pathoanatomical features. Purpose: This study explores myofascial dysfunctions as an alternative explanation to knee pain. Identifying new syndromes termed as knee myofascial pain and knee-abdomen syndromes. $\underline{\text { Therapeutic }}$ intervention: Describing 3 cases of knee osteoarthritis and one case of rheumatoid arthritis treated to full recovery as myofascial dysfunction. All of these cases were investigated and treated to complete recovery from specific myofascial continuity known as deep front line dysfunction, as a cause of knee pain. Results: Both syndromes demonstrated $50 \%$ to $100 \%$ pain reduction after one session of myofascial release, with no recurrence over long-term follow-up after discharge. Conclusion: Knee myofascial pain and knee-abdomen syndromes are clinically present commonly misdiagnosed as arthritic changes. Myofascial release produced an immediate major pain reduction ranging from 50 to $100 \%$. High quality research is required to identify more accurate diagnostic criteria and consequently best treatment strategies.
\end{abstract}

Keywords: Knee, Myofascia, knee-abdomen, Osteoarthritis, Rheumatoid arthritis.

\section{Introduction}

Knee osteoarthritis (OA) is a common diagnosis ${ }^{[1-5]}$, with a reported increased prevalence of $65 \%$ from 1974 to $1994^{[2]}$, and doubling since the mid-20th century ${ }^{[5]}$. OA is a significant contributor to global disability ${ }^{[6]}$, with 303.1 million cases of OA identified globally by $2017^{[6]}$.

Orthopedic medicine diagnostic model depends on radiographic imaging as a cornerstone diagnostic tool ${ }^{[7]}$. Diagnostic pathoanatomical structure identified by imaging are reported in asymptomatic populations; spinal degeneration ,disc bulge and Spinal Cord Compression ${ }^{[8-10]}$, Shoulder rotator cuff tendonitis and tears, labral abnormalities, bursal thickening, and acromioclavicular $\mathrm{OA}^{[11-16]}$, hip's labral tears and cartilage deficit ${ }^{[17,18]}$, femoroacetabular impingement ${ }^{[19]}$, knee's OA, chondral deficits, osteophytes, meniscal tears, tendinopathies ${ }^{[20-25]}$ and bone marrow oedema ${ }^{[26]}$. Knee radiological pathoanatomical findings are present in nearly all (97\%) of asymptomatic/healthy individuals' knees ${ }^{[25]}$, hence our understanding and treatment based on imaging outcomes is misleading. Presence of pathoanatomical features in asymptomatic individuals guided a shift toward dysfunction based diagnostic criteria instead of pathoanatomical ones for patient management of neck and low back pains ${ }^{[27,28]}$. This mandate exploration of causes of knee pain instead of asymptomatically prevalent knee $\mathrm{OA}$ and degenerative changes.

Fascia as a part of the musculoskeletal (MSK) system has the potential to cause pain, dysfunction and disorders ${ }^{[29-34]}$. A multitude of techniques have been created \& utilized by clinicians over the last century targeting this structure ${ }^{[35-40]}$. Fascia remains one of the least studied components of the MSK system. Lack of research regarding myofascial continuities and dysfunction exists, As many anatomists stated that anatomical description has been completed and no new knowledge is to be expected, causing a gradual reduction of budgets and research of anatomy ${ }^{[41]}$. A systematic review of 2016 investigated existence of myofascial lines presented by Tomas Myers in his book (Anatomy trains), concluded that most muscles are connected directly by connective tissue, with evidence regarding the existence of specific myofascial continuities that he termed the superficial back line (SBL), back functional line, and front functional line, these findings can provide a better understanding of musculoskeletal pain and dysfunction development, with a major need for further research ${ }^{[42]}$.

Deep front line (DFL) is a myofascial continuity described in the structural integration text of anatomy trains; authored by Tomas Myers ${ }^{[43]}$, Myers identified an anatomical connection 
between the knee capsule and the abdominal structure through the adductor muscle, medial \& posterior intramuscular septa of the thigh, and Iliopsoas muscle, with its two parts, iliacus and psoas major ${ }^{[43]}$. An explanation of distant structure mutual influence is provided through Biotensegrity ${ }^{[43,44]}$, which is the transmission of a force between connected structures regardless of proximity.

Biotensegrity concept has been studied on the myofascial continuity of the plantar fascia, calf, hamstring and paraspinals, known as the superficial back line ${ }^{[43]}$, with therapeutic based evidence regarding the practical application of Biotensegrity was established, when myofascial release (MFR) was performed on the plantar fascia, an immediate increase of lumbar and hip range of motion was achieved ${ }^{[45]}$, while shortening of calf and hamstring was proven to be associated with plantar fasciitis ${ }^{[46-48]}$. Treatments based on the SBL myofascial continuities was advocated by Myers, and proven effective by research, as stretching of elements of the SBL; the calf and hamstring is determined as an effective treatment for plantar fasciitis ${ }^{[49-51]}$, surgical release of Proximal Medial Gastrocnemius was effective too ${ }^{[52,53]}$.

One issue hindering research and clinical development of manual techniques is descriptive terminology / Nomenclature. Many terms are used within each profession providing manual therapy, as Physical therapy, chiropractic, osteopathy, and others. These techniques are similar linguistically, even though they are indicating different \& diverse techniques, \& theoretical frameworks. This issue led to attempts to unify inter-professions research in spinal manipulation ${ }^{[54]}$. Fascial/myofascial release or manipulation are used interchangeably between clinicians and researchers, with no unification attempts produced yet, hence this paper, uses the term of "myofascial release" to indicate any technique or research regrading either fascial or myofascial manual treatment, while specifying in great details the techniques utilized in treating the presented case studies.

Myofascial release was effective clinically in a multitude of studies of fibromyalgia [55,56], cervical spine dysfunction ${ }^{[57]}$, patellar tendinopathy ${ }^{[58]}$, pubescent postural hyperkyphosis ${ }^{[59]}$, systemic lupus erythematous ${ }^{[60]}$, rotator cuff tear ${ }^{[61]}$, Spastic Cerebral Palsy ${ }^{\left[{ }^{[62]}\right.}$. Even more effective than some standardized physical therapy in cases of low back pain ${ }^{[63]}$. MFR also positively affects the autonomic nervous system's parasympathetic activity with an increase in vagal tone ${ }^{[64,65]}$. An increase in parasympathetic activity can explain the psychological effects of MFR, as improvement of state-trait anxiety ${ }^{[66]}$, and anxiety and depression in fibromyalgia patients ${ }^{[55]}$. Evidence regarding MFR structural integration is lacking quantity because of small sample size and lack of control of some studies ${ }^{[67]}$. This paper present 3 cases that have been encountered with knee pain and medical diagnosis of knee OA and one case of rheumatoid arthritis (RA). All of these cases were investigated and treated to complete recovery from specific myofascial continuity known as deep front line dysfunction, as the cause of knee pain.

Myofascial release was the primary treatment applied to the accessible structure of the patients' DFL, through Myers's MFR techniques of DFL. MFR in its simplest form targeted restoration of mobility of myofascial and fascial layers from within, and with the surrounding structure. MFR techniques utilized with the cases included four variations of application:

1. Muscle was stroked in a longitudinal slow manner, aiming to mobilize fascia over muscles or fascial layers over each other. Which will be referred to as passive release.

2. Separation force applied perpendicularly through a parallel myofascial structure to restore mobility and separation between them. Which will be referred to as separation technique.

3. Force was applied to the myofascial structure while the muscle contracts, aiming to immobilize fascia while muscle contracts and mobilize below it. Which will be referred to as active release.

4. Special form in which combination of the previous is utilized.

The DFL includes the following structures which MFR was targeted to each, tibialis posterior figure ( $1 \& 2)$, toes' long flexors figures ( $3 \& 4)$, knee capsule (no direct release), pes anserine figure (5), adductors of the thigh figure $(6,7 \& 8) \&$ related intramuscular septa figure $(9 \& 10)$, Iliopsoas figure $(11,12 \& 13)$, quadratus lumborum figure (14\&15), diaphragm figure (16), abdominal fascia figure $(17 \& 18)$ and lateral raphe figure (19) ${ }^{[43]}$. Home exercise program consisted of self-stretching of hip adductors, and strengthening of antagonistic muscles of the hip flexors and adductors, specifically the gluteus maximus and medius, to utilize reciprocal control of the tight muscles of the DFL, as advocated by Janda ${ }^{[68]}$, noting that most of the patients became pain free after the first session of MFR, before performing stretching or strengthening exercises, that's utilized as a home exercise program. Utilized MFR techniques are demonstrated on volunteer subject, that provided consent for publication.

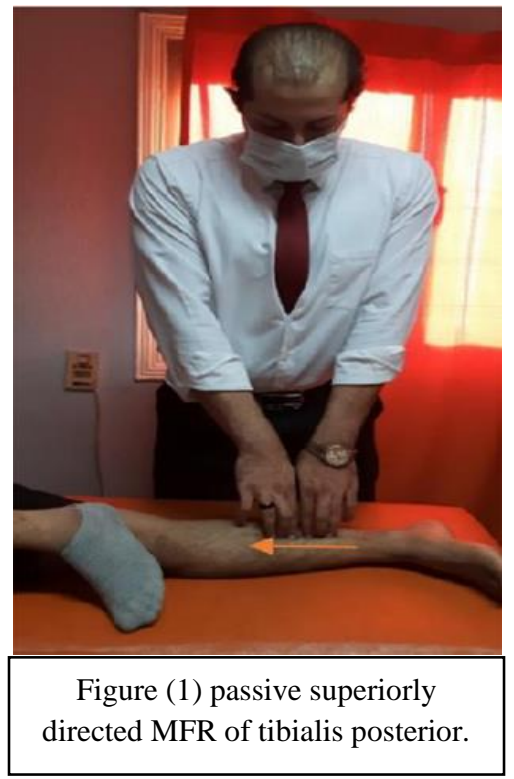

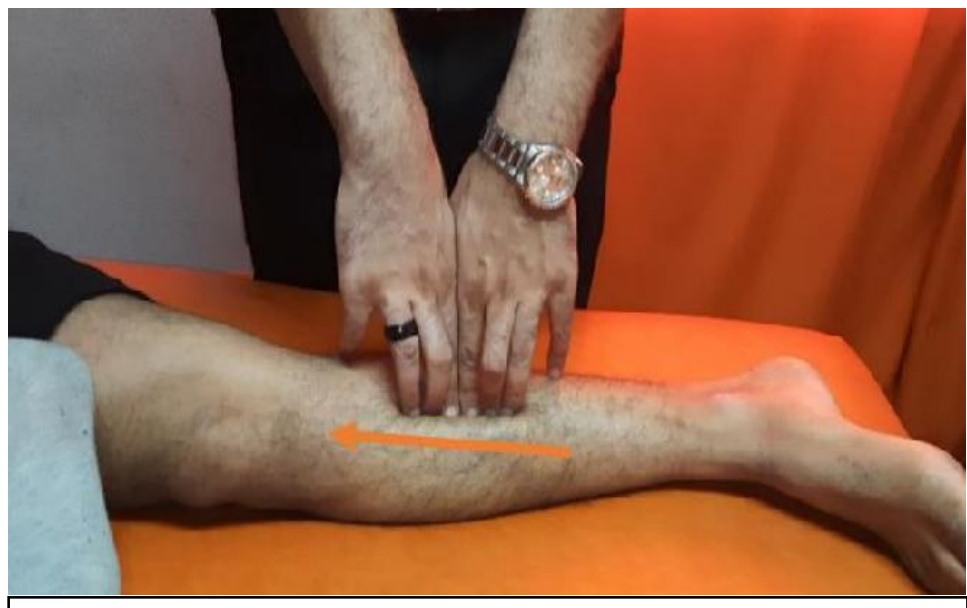

Figure (2) passive superiorly directed MFR of tibialis posterior. 


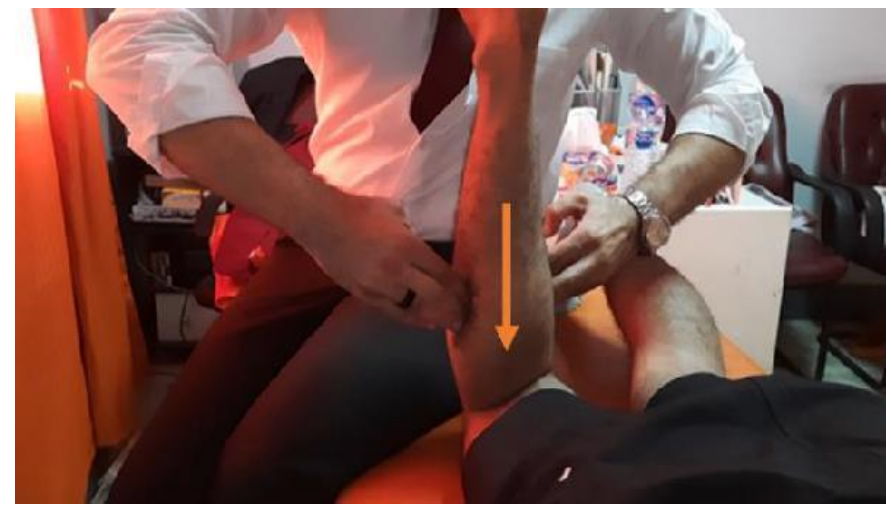

Figure (3) passive superiorly directed MFR of tibialis posterior $\&$ toes' long flexors.

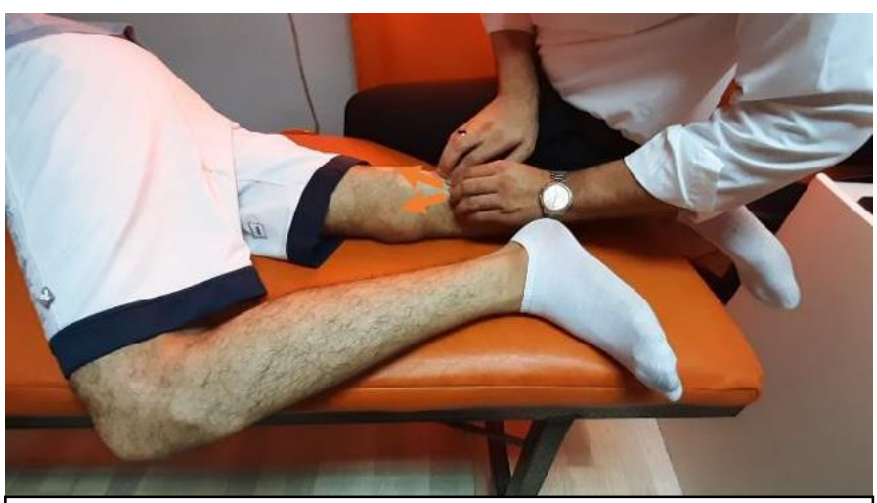

Figure (5) passive superiorly \& laterally directed MFR of pes anserine forming

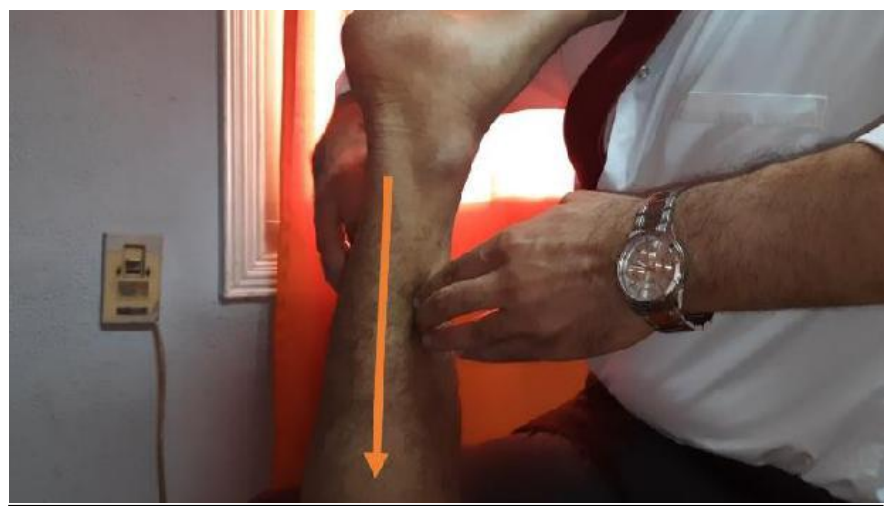

Figure (4) passive superiorly directed MFR of tibialis posterior $\&$ toes' long flexors.

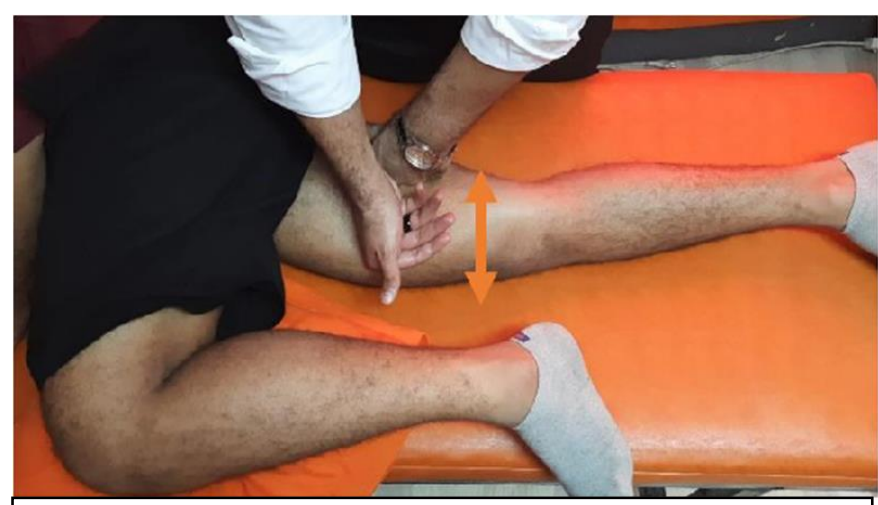

Figure (6) separation technique of hip adductors applied at three hand placements as arrows indicate.
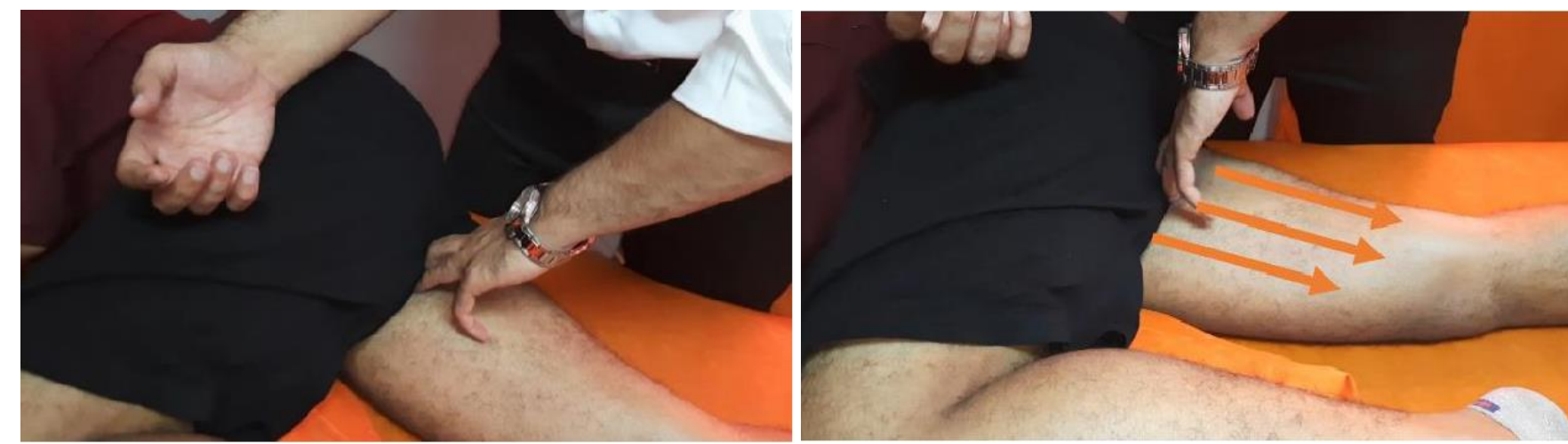

Figure (7\&8) passive inferiorly directed MFR of the adductors at ischio-pubic ramus. Muscles are contacted underneath the ischiopubic ramus at at three hand placements as arrows indicate

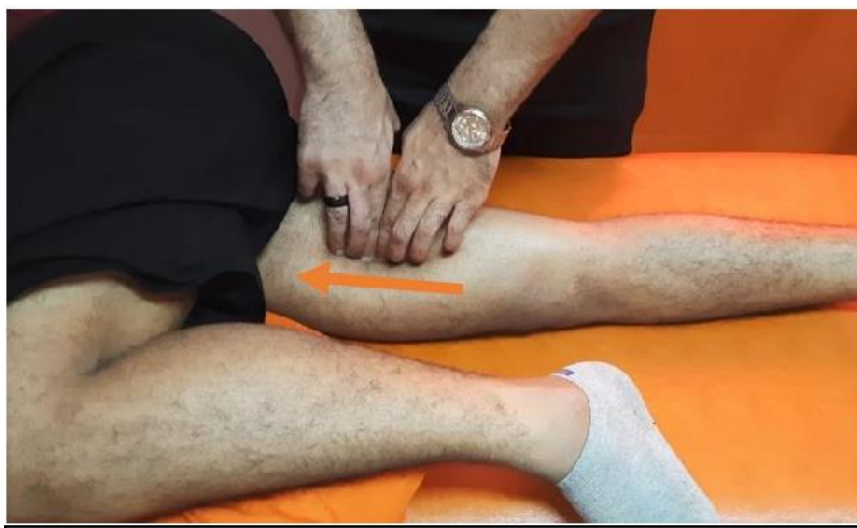

Figure (9) passive superiorly directed MFR of the medial intramuscular septum.

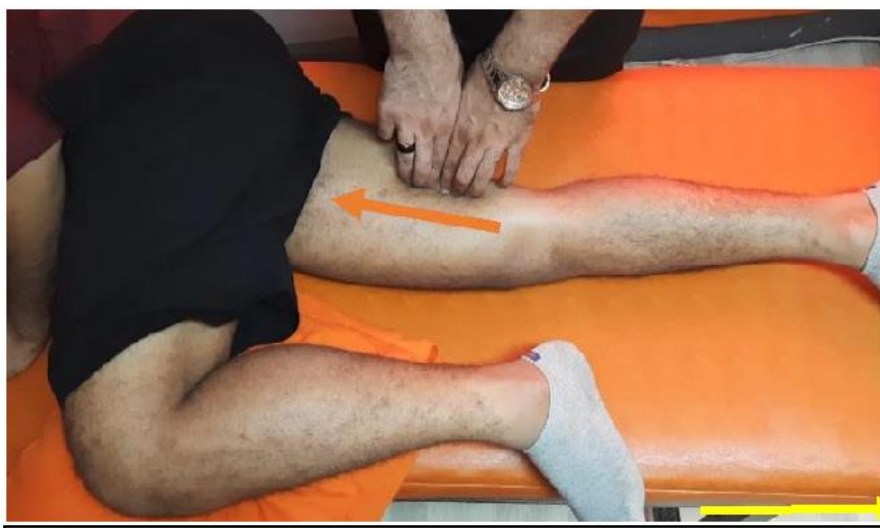

Figure (10) passive superiorly directed MFR of the posterior intramuscular septum. 


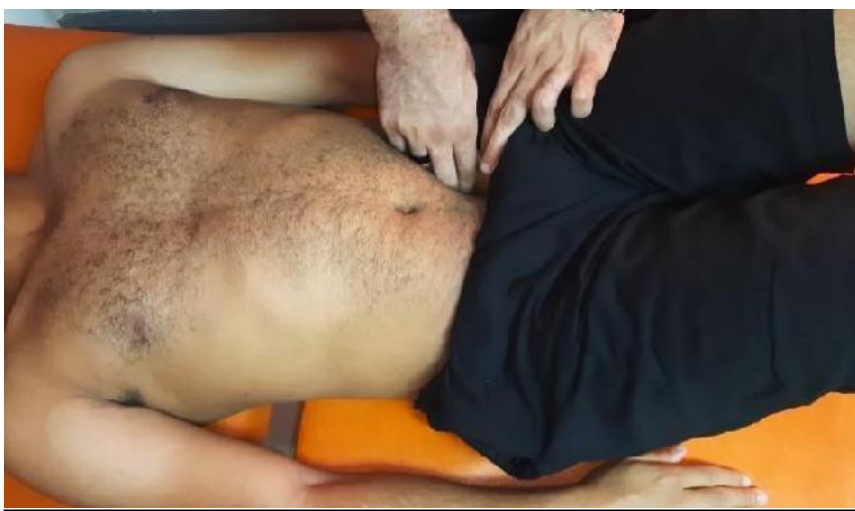

Figure (11) contact point of the iliacus muscle medially to the anterior superior

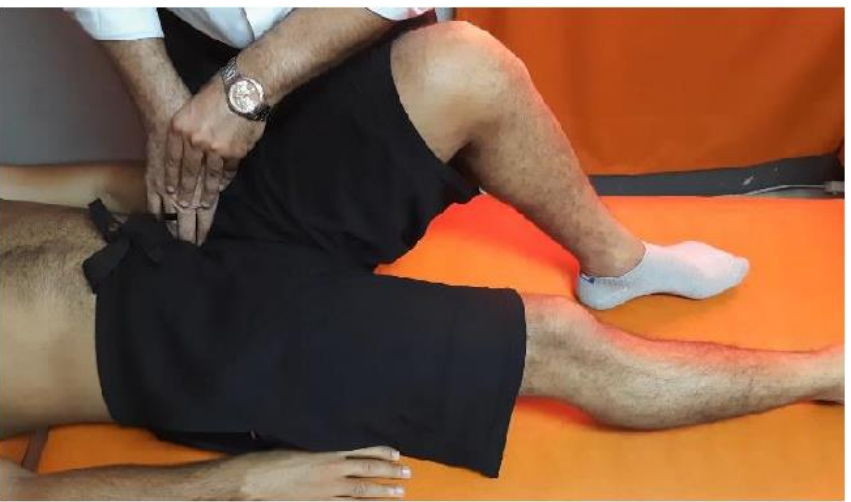

Figure (12) active release of the iliacus, as the iliacus is contacted with pressure $\&$ the patient is instructed to slide his heel \& extend his lower limb.

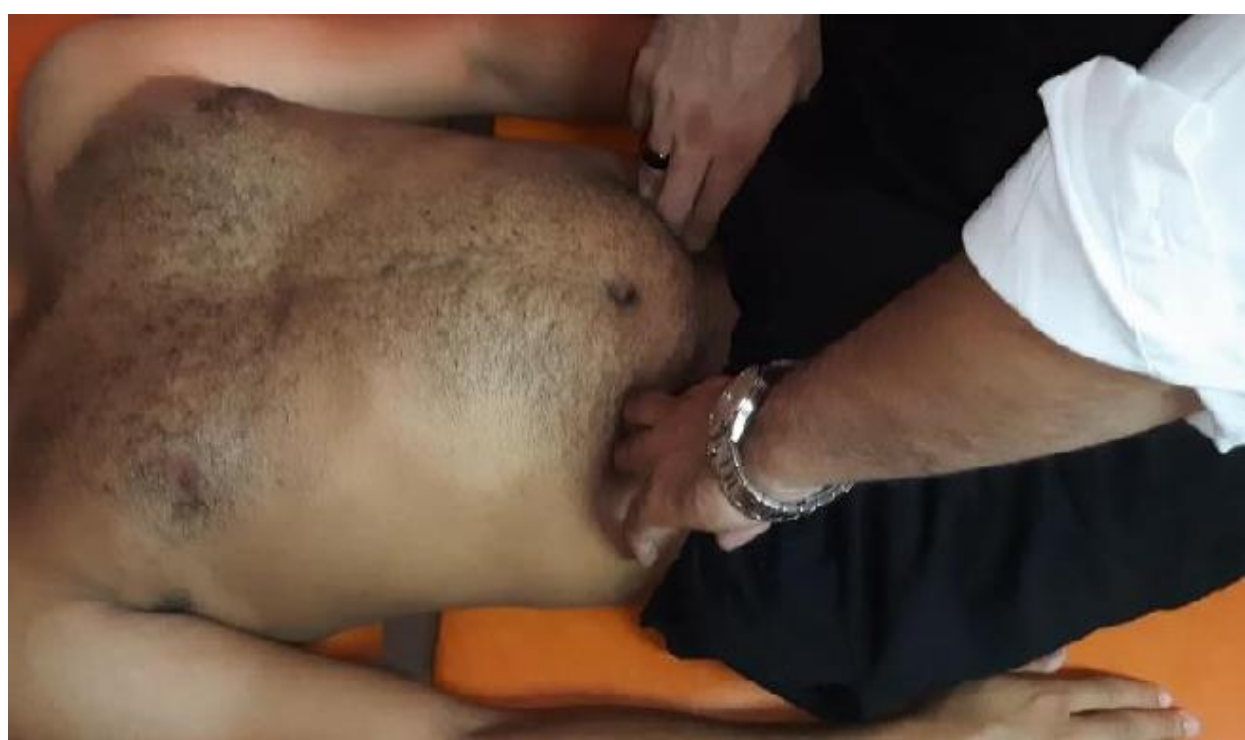

Figure (13) contact point of the psoas major (SM) muscle is medial to the anterior superior iliac with posterior sliding on the inner pelvic surface until contact is achieved with the muscle, confirmed with muscle contraction with active hip flexion \& differentiated from the abdominal wall contraction as a harder \& deeper contraction.

Active release is performed as the patient is instructed to raise their pelvis from the table \& gradually lower their pelvis making gradual contact with each vertebra, while contact with pressure is maintained on SM causing relative

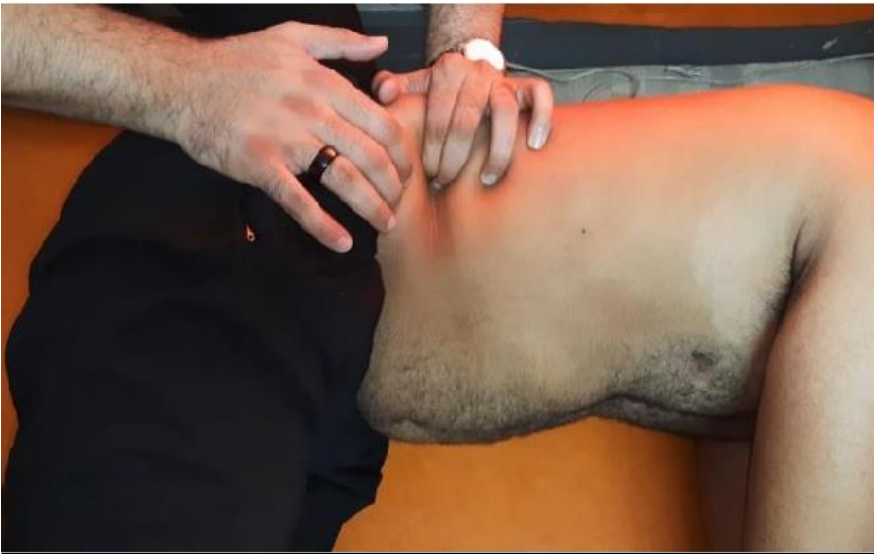

Figure (14) contact point of quadratus lumborum (QL) muscle, through compression of the lateral abdominal wall with posteriorly directed motion, until contact is established with anterior surface of QL.

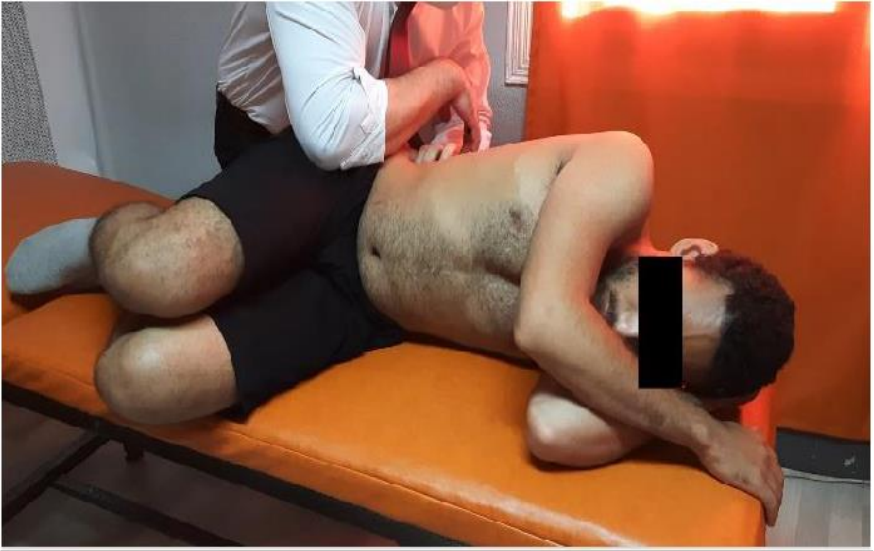

Figure (15) special form release of the QL, as cephalic hand hooks QL \& pulls it posteriorly, maintaining pressure on its anterior surface. the caudal limb forearm brings the pelvis into side bending, lengthening the QL muscular tissue under the relatively immobilized QL's fascia. 


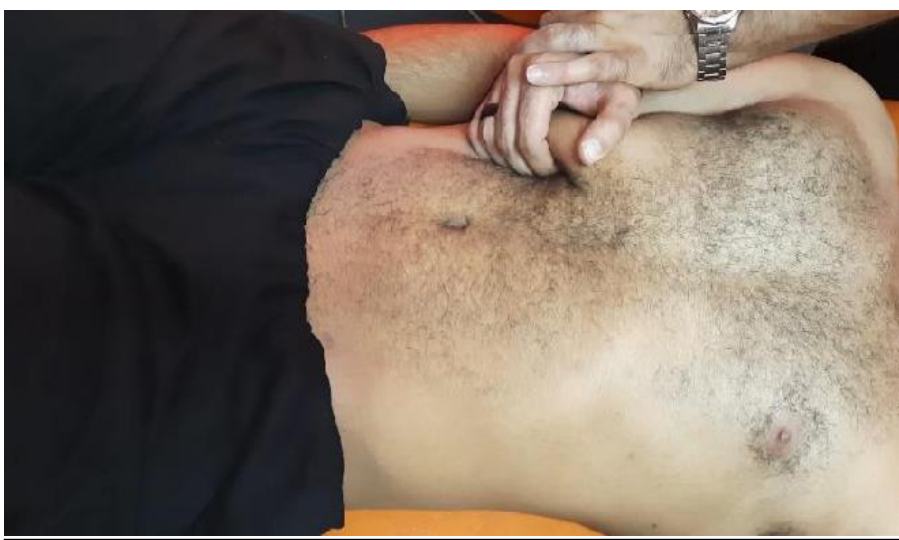

Figure (16) special form release of the diaphragm. Fingers are inserted into the lower costal margin to contact the lowest part of the diaphragmatic myofascia. Ribs pulled slightly laterally to expand the chest $\&$ maintaining relative immobilization contact on the myofascial structure. Patient is instructed to continue breathing regularly for five breaths.

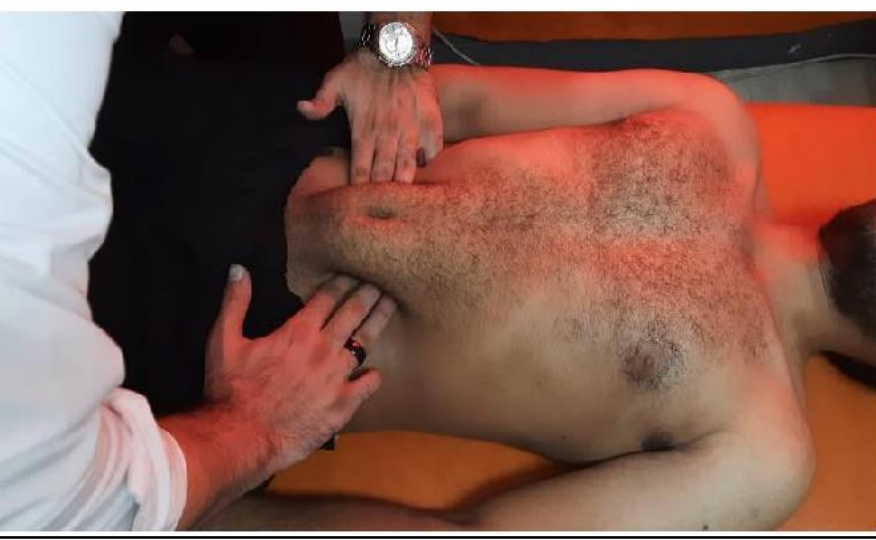

Figure (17) special form release of the abdominal fascia. Fingers are inserted behind the rectus abdominis muscle as deep as possible, with an anteriorly directed lift of the umbilicus is performed, while the patient is instructed to take deep five breaths.

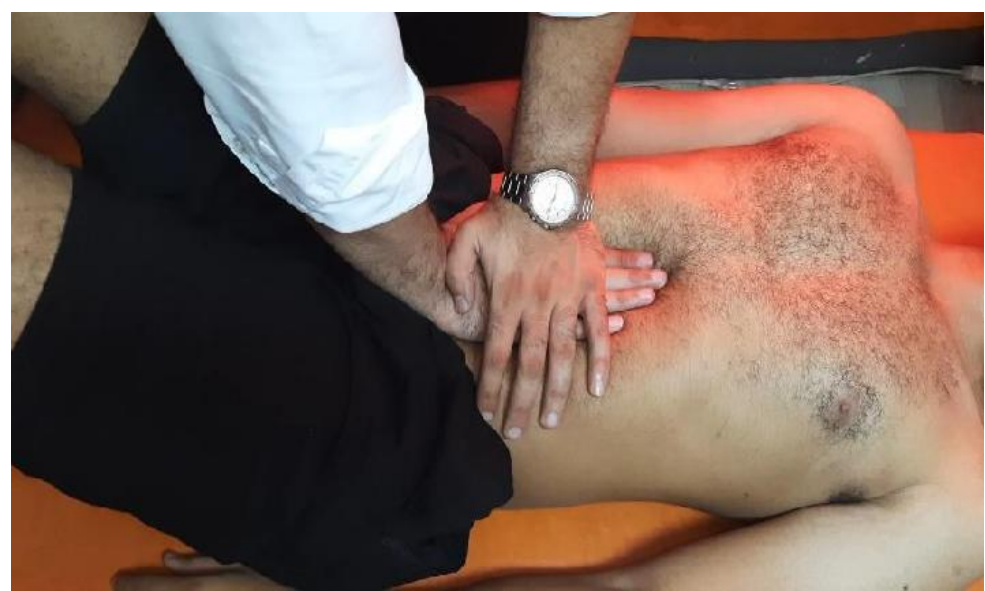

Figure (18) special form release of the abdominal fascia. Hands are placed on the abdomen, maintaining downward force equivalent to the weight of the therapist's upper limbs. Patient is instructed to keep breathing normally, while the position is maintained for 3 minutes. The technique aims to reflect diaphragmatic breathing force to mobilize abdominal fascia.

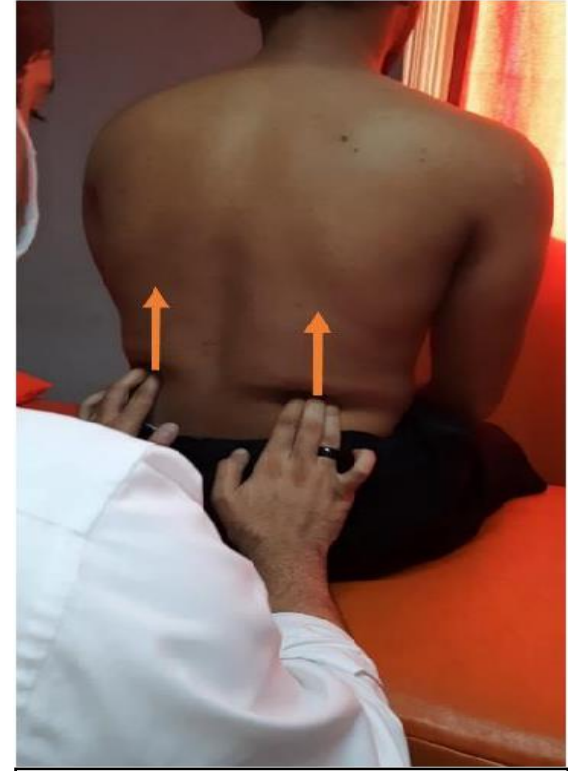

Figure (19) passive superiorly directed MFR of the lateral raphe. Fingers are inserted deeply as the tissue allows superior to the posterior part of the iliac crest, maintaining contact pressure, superior gliding motion is initiated.

\section{Case Study 1}

26 years old female patient complains of bilateral knee pain three months post caesarean section (C-section) delivery. Patient's pain was $6 / 10$ numerical pain rating scale (NRS) ${ }^{[69]}$, exacerbated with weight-bearing flexion as squatting. No clear mechanism of injury with no history of knee pain prior to C-section. Patient demonstrated touch provoked pain over all components of DFL especially hip adductors when compared to other muscle groups, and extreme tenderness on $\mathrm{C}$-section scar line, which friction massage was directed toward it to restore mobility to the scar tissue as part of the treatment. Post one session of MFR pain reduced to $0 / 10$ either in rest or weight-bearing flexion, with no need for other treatment modalities or MFR sessions. 18 months follow-up revealed no recurrence of pain. Patient also complained of low back pain (LBP) that resolved after MFR.

\section{Case Study 2}

63 years old female patient complains of unilateral knee pain with a history of C- section delivery. Patient complains of exacerbated knee pain after stepping into a hole on the beach, patient had knee pain for 20 years, diagnosed as knee OA, modified to OA \& meniscal injury after the steppage incidence.

Patient's pain was $8 / 10$ NRS, exacerbated with weightbearing flexion as squatting or going upstairs. Patient demonstrated touch provoked pain over all components of DFL especially hip adductors when compared to other muscle groups. Patient underwent two treatment sessions of MFR to the DFL. Post 1st session pain was reduced to $2 / 10$ during rest, patient was prescribed a home exercise program (HEP) of strengthening to weak gluteus medius and maximus, and quadriceps, and stretching of hip adductors. 2nd session was 10 days later. Post 2 st session 
pain was reduced to 0/10. Patient was instructed to continue her HEP. Follow-up after 3 month and 1 year revealed no recurrence of pain. To be noted that prior to MFR patient had physical therapy for multiple years with no significant improvement.

\section{Case Study 3}

56 years old female patient complains of LBP with bilateral knee pain. as Knee pain was diagnosed as OA. Patient utilized a handheld cane during walking. Patient's knee pain was 8/10 NRS during rest, exacerbated with weight-bearing flexion as squatting or going upstairs to 10/10. No clear mechanism of injury. Patient demonstrated touch provoked pain over all components of DFL especially hip adductors when compared to other muscle groups. Post one session of MFR pain reduced to 0/10. Patient was prescribed HEP of strengthening for weak gluteus and quadriceps. Patient did not continue treatment as COVID-19 pandemic happened. 8 months follow-up revealed no recurrence of knee symptoms during rest or regular activities of daily living, pain appeared during going upstairs reaching 5/10 NRS, with cessation of cane use as a walking aid. Patient's low back pain reduction was reported.

\section{Case Study 4}

58 years old female patient complains of unilateral knee pain, diagnosed with RA, presented with flexion deformation with an inability to extend the knee over $20^{\circ}$ flexion, either in weightbearing or non-weight bearing, with $8 / 10$ pain during standing reaching 10/10 with active or passive full knee extension force. Patient reported pain variation was enough severity to prevent sleeping at some nights. Patient demonstrated touch provoked pain over all components of DFL especially hip adductors when compared to other muscle groups. Patient underwent 11 sessions of PT, once per week. 1st session treatment included MFR of DFL \& strengthening exercise of the weakened gluteus medius and maximus, and quadriceps muscle, and stretching of hip adductors. Directly after MFR pain reached $0 / 10$ of non-weight-bearing and $3 / 10$ in weight-bearing with inability to perform full knee extension, exercise did not alter pain levels. Patient was prescribed HEP of strengthening exercise to the forementioned muscle, stretch to hip's adductors and heel prop to increase knee extension. After 4 session of MFR and strengthening HEP patient achieved 0/10 nonweight and weight- bearing, pain increased with standing or walking over 15 minutes, and reduced with rest and medications, the same medication was reported to have a much lesser effect on pain before. With no improvement in knee extension. As pain was controlled 5th session targeted increase of knee's extension range of motion (ROM) with mobilization and stretching exercise, pain levels increased with mobilization to reach $6 / 10$ of non-weightbearing and 8/10 in weight-bearing. The 6th session included MFR targeting pain reduction, which caused $0 / 10$ pain outcome, also introduced balance exercises. The following 5 sessions alternated between increasing ROM \& reducing pain. After 11 sessions pt achieved $0 / 10$ either in non-weight or weight-bearing with full knee extension range of motion. Pain occurs after hour of standing or walking. Knee pain had not prevented sleep over the last 2 weeks of therapy. Patient was discharged from PT as no further improvement has been achieved, with an advice of lifetime maintenance of her prescribed HEP. 3 months follow-up revealed no regression of the case or reappearance of pain. Patient's low back pain reduction was also reported.
Patient received physical therapy before with pain improvement that never reached $0 / 10$ or complete knee extension, and NSAIDs had minimal effect on pain. By contacting her previous physical therapists, knee joint mobilization and exercise were too painful and performed withing tolerance but still knee extension was never achieved. By the 4th month after discharge patient reported to the physical therapy clinic with reappearance of knee pain with limitation of knee extension. Patient had one month of vacation when she stopped her HEP, after 2 weeks of cessation of HEP pain reappeared and progressed rapidly, by the time of this article was written patient restarted Physical Therapy with pain of $8 / 10$ during standing. Post one session patient pain reduced to $4 / 10$ with improved knee extension, and she'll continue receiving the same previous treatment.

\section{Discussion}

All presented cases demonstrated major pain reduction on NRS ranging from $50 \%$ to $100 \%$ after one treatment. Case 1 demonstrated tenderness to C-section scar line, \& complete resolution of pain from MFR with no need for further treatment or HEP. Cases 2 and 4 required more than one treatment session with concomitant muscle imbalance that required directed exercise. All of the 4 cases are termed to have knee myofascial pain syndrome, while case 1 had a sub-classification of knee myofascial pain syndrome to be termed knee-abdomen syndrome, as knee pain's primary cause is abdominal fascia incision of $\mathrm{C}$-section through DFL.

All patients demonstrated touch provoked pain over all components of DFL when compared to other muscle groups, especially hip adductors, this pain was not identified by the patient until touch provoked it. This finding is the only present symptom of both syndromes, that to can be used diagnostically prior to MFR, the only sure sign is major pain reduction following MFR.

The four presented cases are representations of different patterns of knee pain of myofascial origin. In case 1 deep front line dysfunction (DFLD) was a standalone dysfunction, while in cases 2 and3 DFLD was associated with muscle imbalance, $\&$ in case 4 was associated with muscle imbalance and RA. The three recurring patterns are observed repeatedly clinically, as the most common pattern is DFLD with muscle imbalance especially weakness of gluteus medius and maximus, and quadriceps. Weakness of the gluteus medius and maximus can be attributed to tightness inhibition relation ${ }^{[68]}$, as tightness of specific muscle can reciprocally inhibit its antagonist and vice versa. Whether tightness of adductors or iliopsoas muscles caused weakness of gluteus medius and maximus, or weakness of the muscles caused tightness and dysfunction of the DFL is unknown to the authors. Sound clinical judgment requires managing both components as patient's treatment, whether one treatment of exercise alone or MFR alone well be enough to treat patients to full recovery is unknown \& untested.

The second identified pattern is less common \& encountered only twice by the authors, in which DFLD rises as a complication to C-section, \& treated to 0/10 NRS within one session. The third pattern coexistence of DFLD and muscle imbalance with RA, in which treatment was required to all three components. Medications for RA with MFR and exercise.

Within the three presented patterns, standalone DFLD required minimal duration treatment of MFR alone, demonstrating immediate and long last recovery with no need for further treatment. DFLD with muscle imbalance required combined treatment with multiple sessions \& HEP. After the disappearance 
of pain and normalization of muscle strength pain did not recure during long-term follow-up. The third pattern of DFLD with muscle imbalance and RA demonstrated slowest recovery rate with recurrence of symptoms with cessation of physical activity and HEP.

This scientific paper represents a general call to the biomedical community to start a widespread research of the presented syndromes and the most efficient and effective treatment strategies, and subsequent changes to $\mathrm{OA}$ diagnostic criteria, as well as generation of diagnostic criteria for knee-abdomen and knee myofascial pain syndromes.

\section{Conclusions}

Knee myofascial pain syndrome and knee-abdomen syndrome are newly identified causes of knee pain usually misdiagnosed as knee OA or arthritic changes. Knee-abdomen syndrome demonstrated complete resolution after one treatment session with no recurrence over long-term follow-up and is defined as DFL myofascial dysfunction with a direct cause of abdominal fascia incision. Knee myofascial pain syndrome is a DFL myofascial dysfunction associated with muscle imbalance that required addition of therapeutic exercises to MFR, with multiple sessions of treatment. Both syndromes demonstrated $50 \%$ to $100 \%$ pain reduction after one session of MFR, with no recurrence over long-term follow-up after discharge, unless another active cause was present as RA. Patients demonstration of touch provoked pain over components of DFL is the only present symptom of both syndromes, that can be used diagnostically prior to MFR. High quality research is required to identify more accurate diagnostic criteria and best treatment strategies.

\section{Ethics approval and consent to participate}

The presented cases were not preplanned as a clinical study. This paper represents reporting of clinical cases suspected \& treated as myofascial dysfunction; hence no ethical committee approval was sought. Each case had an explanation regarding possibility of myofascial dysfunction contributing to her Knee pain, and verbal consent were given prior to MFR

\section{List of abbreviations}

Knee osteoarthritis (OA)

Musculoskeletal (MSK)

Superficial back line (SBL)

Deep front line (DFL)

Myofascial release (MFR)

Numerical pain rating scale (NRS)

Low back pain (LBP)

Deep front line dysfunction (DFLD)

\section{Conflicts of Interest}

The authors declare that there is no conflict of interest regarding the publication of this paper

\section{Funding Statement}

No funding was needed

\section{Authors' contributions}

I.T identified \& performed clinical treatments, researched \& cowritten the article.

O.K researched \& edited the article

\section{References}

[1] Felson, D.T., et al., The prevalence of knee osteoarthritis in the elderly. The Framingham Osteoarthritis Study. Arthritis \& Rheumatism: Official Journal of the American College of Rheumatology, 1987. 30(8): p. 914918.

[2] Nguyen, U.-S.D., et al., Increasing prevalence of knee pain and symptomatic knee osteoarthritis: survey and cohort data. Annals of internal medicine, 2011. 155(11): p. 725-732.

[3] Heidari, B., Knee osteoarthritis prevalence, risk factors, pathogenesis and features: Part I. Caspian journal of internal medicine, 2011. 2(2): p. 205.

[4] Jordan, J.M., et al., Prevalence of knee symptoms and radiographic and symptomatic knee osteoarthritis in African Americans and Caucasians: the Johnston County Osteoarthritis Project. The Journal of rheumatology, 2007. 34(1): p. 172-180.

[5] Wallace, I.J., et al., Knee osteoarthritis has doubled in prevalence since the mid-20th century. Proceedings of the National Academy of Sciences, 2017. 114(35): p. 9332-9336.

[6] Safiri, S., et al., Global, regional and national burden of osteoarthritis 1990-2017: a systematic analysis of the global burden of disease study 2017. Annals of the rheumatic diseases, 2020. 79(6): p. 819-828.

[7] Nayagam, S., D. Warwick, and L. Solomon, Apley's system of orthopaedics and fractures. 2010: Hodder Arnold.

[8] Brinjikji, W., et al., Systematic literature review of imaging features of spinal degeneration in asymptomatic populations. American Journal of Neuroradiology, 2015. 36(4): p. 811-816.

[9] Van den Heuvel, M.M., et al., The prevalence of abnormalities in the pediatric spine on MRI: a systematic review and meta-analysis. Spine, 2020. 45(18): p. E1185-E1196.

[10] Smith, S.S., et al., The prevalence of asymptomatic and symptomatic spinal cord compression on magnetic resonance imaging: a systematic review and metaanalysis. Global Spine Journal, 2020: p. 2192568220934496.

[11] Miniaci, A., et al., Magnetic resonance imaging evaluation of the rotator cuff tendons in the asymptomatic shoulder. The American journal of sports medicine, 1995. 23(2): p. 142-145.

[12] Girish, G., et al., Ultrasound of the shoulder: asymptomatic findings in men. American Journal of Roentgenology, 2011. 197(4): p. W713-W719.

[13] Gill, T.K., et al., Prevalence of abnormalities on shoulder MRI in symptomatic and asymptomatic older adults. International journal of rheumatic diseases, 2014. 17(8): p. 863-871.

[14] Barreto, R.P.G., et al., Bilateral magnetic resonance imaging findings in individuals with unilateral shoulder pain. Journal of shoulder and elbow surgery, 2019. 28(9): p. 1699-1706. 
[15] Frigg, A., et al., Seven-year course of asymptomatic acromioclavicular osteoarthritis diagnosed by MRI. Journal of shoulder and elbow surgery, 2019. 28(10): p. e344-e351.

[16] Hacken, B., et al., Prevalence of MRI Shoulder Abnormalities in Asymptomatic Professional and Collegiate Ice Hockey Athletes. Orthopaedic journal of sports medicine, 2019. 7(10): p. 2325967119876865.

[17] Heerey, J.J., et al., What is the prevalence of imagingdefined intra-articular hip pathologies in people with and without pain? A systematic review and meta-analysis. British Journal of Sports Medicine, 2018. 52(9): p. 581593.

[18] Heerey, J.J., et al., What is the prevalence of hip intraarticular pathologies and osteoarthritis in active athletes with hip and groin pain compared with those without? A systematic review and meta-analysis. Sports Medicine, 2019. 49(6): p. 951-972.

[19] Mascarenhas, V.V., et al., Imaging prevalence of femoroacetabular impingement in symptomatic patients, athletes, and asymptomatic individuals: a systematic review. European journal of radiology, 2016. 85(1): p. 73-95.

[20] Flanigan, D.C., et al., Prevalence of chondral defects in athletes' knees: a systematic review. Medicine \& Science in Sports \& Exercise, 2010. 42(10): p. 1795-1801.

[21] Pappas, G.P., et al., Magnetic resonance imaging of asymptomatic knees in collegiate basketball players: the effect of one season of play. Clinical journal of sport medicine: official journal of the Canadian Academy of Sport Medicine, 2016. 26(6): p. 483.

[22] Beals, C.T., et al., The prevalence of meniscal pathology in asymptomatic athletes. Sports Medicine, 2016. 46(10): p. 1517-1524.

[23] Keng, A., et al., Association of body mass index with knee cartilage damage in an asymptomatic populationbased study. BMC Musculoskeletal Disorders, 2017. 18(1): p. 1-6.

[24] Culvenor, A.G., et al., Prevalence of knee osteoarthritis features on magnetic resonance imaging in asymptomatic uninjured adults: a systematic review and meta-analysis. British journal of sports medicine, 2019. 53(20): p. 12681278.

[25] Horga, L.M., et al., Prevalence of abnormal findings in 230 knees of asymptomatic adults using 3.0 T MRI. Skeletal radiology, 2020. 49(7): p. 1099-1107.

[26] Matiotti, S.B., et al., MRI of the knees in asymptomatic adolescent soccer players: a case control study. Journal of Magnetic Resonance Imaging, 2017. 45(1): p. 59-65.

[27] Delitto, A., et al., Low back pain: clinical practice guidelines linked to the international classification of functioning disability, and health from the orthopaedic section of the American Physical Therapy Association. Journal of orthopaedic and sport physical therapy, 2012. 42(4).

[28] Blanpied, P.R., et al., Neck pain: revision 2017: clinical practice guidelines linked to the international classification of functioning, disability and health from the orthopaedic section of the American Physical Therapy Association. Journal of Orthopaedic \& Sports Physical Therapy, 2017. 47(7): p. A1- A83.
[29] Stecco, C., et al., Anatomy of the deep fascia of the upper limb. Second part: study of innervation. Morphologie, 2007. 91(292): p. 38-43.

[30] Stecco, C., et al., Hyaluronan within fascia in the etiology of myofascial pain. Surgical and radiologic anatomy, 2011.33(10): p. 891-896.

[31] Stecco, A., et al., Fascial components of the myofascial pain syndrome. Current pain and headache reports, 2013. 17(8): p. 352.

[32] Schleip, R., W. Klingler, and F. Lehmann-Horn. Active contraction of the thoracolumbar fascia indications of a new factor in low back pain research with implications for manual therapy. in Proceedings of the Fifth Interdisciplinary World Congress on Low Back and Pelvic Pain. Melbourne, Australia: Fascia Research Project. 2004.

[33] Schleip, R., A. Zorn, and W. Klingler, Biomechanical properties of fascial tissues and their role as pain generators. Journal of musculoskeletal pain, 2010. 18(4): p. 393-395.

[34] Klingler, W., et al., Clinical relevance of fascial tissue and dysfunctions. Current pain and headache reports, 2014. 18(8): p. 439.

[35] Manheim, C.J., Myofascial Release Manual, the: Carol J Manheim; editor: Cheryl D Willoughby. 1989.

[36] Kostopoulos, D. and K. Rizopoulos, The manual of trigger point and myofascial therapy. 2001: Slack.

[37] Hunter, G., Myofascial Manipulation Theory and clinical application. Physiotherapy, 2001. 87(9): p. 501-502.

[38] Stecco, L., J.V. Basmanjian, and J.A. Day, Fascial manipulation for musculoskeletal pain. 2004: Piccin Padova.

[39] Nicholas, A.S. and E.A. Nicholas, Atlas of osteopathic techniques. 2008: Lippincott Williams \& Wilkins.

[40] Duncan, R., Myofascial release. 2014: Human Kinetics.

[41] Huijing, P.A., Epimuscular myofascial force transmission: a historical review and implications for new research. International Society of Biomechanics Muybridge Award Lecture, Taipei, 2007. Journal of biomechanics, 2009. 42(1): p. 9-21.

[42] Wilke, J., et al., What is evidence-based about myofascial chains: a systematic review. Archives of physical medicine and rehabilitation, 2016. 97(3): p. 454461.

[43] Myers, T., Anatomy Trains, Myofascial Meridians for Manual and Movement Therapists 2nd edition, Churchill Livingstone. 2009, Elsevier Edinburgh.

[44] Levin, S.M. and D.-C. Martin, Biotensegrity: the mechanics of fascia. Fascia e the Tensional Network of the Human Body. The Science and Clinical Applications in Manual and Movement Therapy. Elsevier, Edinburgh, 2012: p. 137-142.

[45] Do, K., J. Kim, and J. Yim, Acute effect of selfmyofascial release using a foam roller on the plantar fascia on hamstring and lumbar spine superficial back line flexibility. Physical Therapy Rehabilitation Science, 2018. 7(1): p. 35-40.

[46] Patel, A. and B. DiGiovanni, Association between plantar fasciitis and isolated contracture of the gastrocnemius. Foot \& ankle international, 2011. 32(1): p. 5-8. 
[47] Labovitz, J.M., J. Yu, and C. Kim, The role of hamstring tightness in plantar fasciitis. Foot \& ankle specialist, 2011. 4(3): p. 141-144.

[48] Bolívar, Y.A., P.V. Munuera, and J.P. Padillo, Relationship between tightness of the posterior muscles of the lower limb and plantar fasciitis. Foot \& ankle international, 2013. 34(1): p. 42-48.

[49] Engkananuwat, P., R. Kanlayanaphotporn, and N. Purepong, Effectiveness of the simultaneous stretching of the Achilles tendon and plantar fascia in individuals with plantar fasciitis. Foot \& ankle international, 2018. 39(1): p. 75-82.

[50] Garrett, T.R. and P.J. Neibert, The effectiveness of a Gastrocnemius-Soleus stretching program as a therapeutic treatment of plantar fasciitis. Journal of sport rehabilitation, 2013. 22(4): p. 308-312.

[51] Kamonseki, D.H., et al., Effect of stretching with and without muscle strengthening exercises for the foot and hip in patients with plantar fasciitis: a randomized controlled single-blind clinical trial. Manual therapy, 2016. 23: p. 76-82.

[52] Abbassian, A., J. Kohls-Gatzoulis, and M.C. Solan, Proximal medial gastrocnemius release in the treatment of recalcitrant plantar fasciitis. Foot \& ankle international, 2012. 33(1): p. 14-19.

[53] Monteagudo, M., et al., Chronic plantar fasciitis: plantar fasciotomy versus gastrocnemius recession. International orthopaedics, 2013. 37(9): p. 1845-1850.

[54] Mintken, P.E., et al., A model for standardizing manipulation terminology in physical therapy practice. journal of orthopaedic \& sports physical therapy, 2008. 38(3): p. A1-A6.

[55] Stall, P. and M.J. Teixeira, Fibromyalgia syndrome treated with the structural integration Rolfing ${ }^{\circledR}$ method. Revista Dor, 2014. 15(4): p. 248-252.

[56] Castro-Sánchez, A.M., et al., Effects of myofascial release techniques on pain, physical function, and postural stability in patients with fibromyalgia: a randomized controlled trial. Clinical Rehabilitation, 2011. 25(9): p. 800-813.

[57] James, H., et al., Rolfing structural integration treatment of cervical spine dysfunction. Journal of Bodywork and Movement Therapies, 2009. 13(3): p. 229-238.

[58] Pedrelli, A., C. Stecco, and J.A. Day, Treating patellar tendinopathy with Fascial Manipulation. Journal of bodywork and movement therapies, 2009. 13(1): p. 7380.

[59] Ćosić, V., et al., Fascial Manipulation ${ }^{\circledR}$ method applied to pubescent postural hyperkyphosis: A pilot study. Journal of bodywork and movement therapies, 2014. 18(4): p. 608-615.

[60] Ball, T.M., Structural integration-based fascial release efficacy in systemic lupus erythematosus (SLE): two case studies. Journal of Bodywork and Movement Therapies, 2011. 15(2): p. 217-225.

[61] Pintucci, M., et al., Successful treatment of rotator cuff tear using Fascial Manipulation ${ }^{\circledR}$ in a stroke patient.
Journal of Bodywork and Movement Therapies, 2017. 21(3): p. 653-657.

[62] Hansen, A.B., K.S. Price, and H.M. Feldman, Myofascial structural integration: a promising complementary therapy for young children with spastic cerebral palsy. Journal of Evidence-Based Complementary \& Alternative Medicine, 2012. 17(2): p. 131-135.

[63] Harper, B., L. Steinbeck, and A. Aron, Fascial manipulation vs. standard physical therapy practice for low back pain diagnoses: A pragmatic study. Journal of Bodywork and Movement Therapies, 2019. 23(1): p. 115-121.

[64] Cottingham, J.T., S.W. Porges, and K. Richmond, Shifts in pelvic inclination angle and parasympathetic tone produced by Rolfing soft tissue manipulation. Physical Therapy, 1988. 68(9): p. 1364-1370.

[65] Cottingham, J.T., S.W. Porges, and T. Lyon, Effects of soft tissue mobilization (Rolfing pelvic lift) on parasympathetic tone in two age groups. Physical therapy, 1988. 68(3): p. 352-356.

[66] Weinberg, R.S. and V.V. Hunt, Effects of structural integration on state- trait anxiety. Journal of Clinical Psychology, 1979. 35(2): p. 319-322.

[67] Jacobson, E., Structural integration, an alternative method of manual therapy and sensorimotor education. The Journal of Alternative and Complementary Medicine, 2011. 17(10): p. 891-899.

[68] Page, P., C.C. Frank, and R. Lardner, Assessment and treatment of muscle imbalance: the Janda approach. 2010: Human kinetics Champaign, IL.

[69] Alghadir, A.H., et al., Test-retest reliability, validity, and minimum detectable change of visual analog, numerical rating, and verbal rating scales for measurement of osteoarthritic knee pain. Journal of pain research, 2018. 11: p. 851 . 